Gut, 1962, 3, 306

\title{
The management of fulminant ulcerative colitis
}

\author{
N. D. GALlAGHER, S. J. M. GOULSTON, N. WYNDHAM, \\ AND SIR WILLIAM MORROW \\ From the Gastroenterology Unit, Royal Prince Alfred Hospital, Sydney, Australia
}

EDITORIAL SYNOPSIS This paper describes the management of 23 patients with fulminant ulcerative colitis, 22 of whom had early surgery when medical treatment was unsuccessful. There were two deaths, a mortality of $9 \%$.

The small number of patients with ulcerative colitis who enter a fulminant phase remain a difficult problem in management. We have previously defined the illness as a grave, explosive episode in the course of non-specific ulcerative colitis, with high fever, tachycardia, extreme toxaemia, rapid loss of weight, and varying degrees of diarrhoea, rectal haemorrhage, and abdominal tenderness and distension (Rankin, Goulston, Boden, and Morrow, 1960). Its severity is such that few patients survive with supportive measures alone. Treatment with corticosteroids or corticotrophin may also be of little benefit and cause unnecessary delay in advising surgery (Lennard-Jones and Vivian, 1960; Rankin et al., 1960). Prolonged supportive and corticosteroid therapy or delayed surgery were associated with a mortality of $61 \%$ or 11 deaths in 18 patients treated by us in the period 1950-58. In an attempt to lessen mortality it was decided that colectomy should be recommended at an early stage of the illness if no sustained improvement occurred with conservative therapy. This paper reports our experience with 23 consecutive patients with fulminant colitis, only one of whom did not require surgery. There were two deaths, a mortality of $9 \%$.

\section{CLINICAL FEATURES}

The clinical features of the group will be briefly described. Details of age, sex, and laboratory investigations are shown in Table I. The fulminant disease arose as the initial illness in five patients and during the course of chronic ulcerative colitis in 18 patients. In three of the 18 patients the disease had begun as a local proctosigmoiditis. Factors which may have precipitated the illness were uncertain but three had major emotional problems at the time the illness began and one had a relapse which coincided with an attack of infectious mononucleosis.
The invariable complaint was severe diarrhoea. Incontinence or rectal prolapse occasionally occurred. Stool volumes were very large, 1,500 to $3,000 \mathrm{ml}$. being passed daily. Brisk haemorrhage occurred in two patients and blood was generally present in the stools of the remainder. All patients became very distressed during the illness and confusion, apathy, euphoria, or depression were common. Fever and tachycardia were always present. Loss of appetite was marked and weight loss severe. Abdominal pain and tenderness were frequently noted. Eight patients or one third of the group developed gross abdominal distension. Severe xeroderma was present in two patients and three had cirrhosis of the liver.

\section{INVESTIGATIONS}

The results of laboratory investigations are shown in Table I. The values shown are the lowest found during the illness with the exception of the white cell count for which the highest values are shown. Anaemia was generally present, although the degree was often masked by haemoconcentration. White cell counts were as high as 42,000 cells/c.mm. and neutrophil band forms or unclassifiable cells were noted frequently. Lowered serum concentrations of sodium and potassium were found in the majority and a marked reduction in serum albumin or total protein was the rule. Microscopic examination of the stools revealed profuse numbers of red and white blood cells and no evidence of cysts, ova, or parasites. $E$. coli and $B$. proteus were usually grown from stool culture. Sigmoidoscopic examination showed the appearances of ulcerative colitis in a mild or severe form but was normal in one patient. A plain $x$-ray examination of the abdomen demonstrated marked colonic dilatation in eight patients. A second examination after a 10-day interval in a patient 
TABLE I

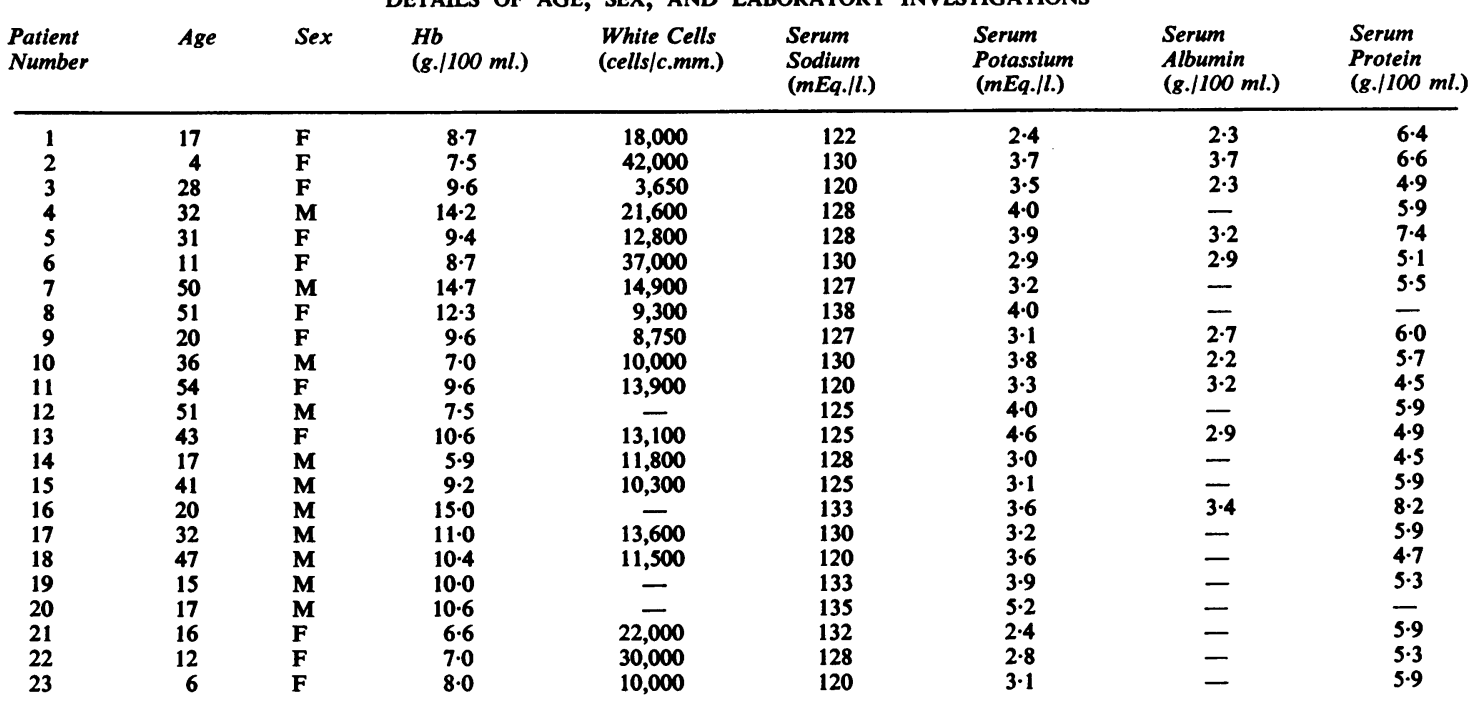

without colonic dilatation showed numerous polyps. Fourteen patients had a barium enema examination. The appearances in six were those of chronic ulcerative colitis with changes such as loss of haustrations, rigidity of the colonic wall, and pseudo-polyp formation. The examination in the remaining eight patients was more suggestive of an acute ulcerative process with variable loss of haustrations and irregularity of the colonic outline.

\section{PATHOLOGY}

Widespread longitudinal or transverse ulceration was found in 21 of the 22 colons examined. Examination of the remaining colon revealed intense congestion and oedema of all coats. The ulcers were of the deep variety, extending into the muscle coat. The surviving mucosa was inflamed and oedematous. Pseudo-polyps, which were usually the result of undermining of the mucosa adjacent to the ulcer, were common. Examination of the vessels was normal.

\section{MANAGEMENT AND RESULTS}

The aim in management was to provide full supportive therapy for a period of one to two weeks, depending on the rate of progression of the disease, and to recommend surgery if no significant improvement occurred.

All patients received intensive supportive therapy consisting of intravenous transfusion of whole blood and albumin, sodium and potassium chloride, and glucose, iron, and vitamins. During this period they were kept in bed and given every possible form of encouragement. Oral feeding was permitted but anorexia was such that it made an insignificant contribution to daily intake. Strict fluid balance was maintained and close attention to intravenous therapy ensured that replacement therapy was proceeding as planned. Frequent estimations of haemoglobin, serum electrolytes, and serum proteins were performed. A careful assessment of changes in the severity of the illness was made in the morning and evening of each day. When it appeared that surgery would be necessary, early consultation with the surgeon and anaesthetist was arranged. Antibiotic therapy was usually confined to the 48-hour period before surgery when intravenous tetracyclines and oral neomycin were administered.

The benefits of supportive and antibiotic therapy were often strikingly apparent in an increase in well being and decline in fever and tachycardia but persistent diarrhoea, unchanging abdominal signs, and progressive decrease in haemoglobin, serum protein, and serum electrolyte concentrations indicated that there had been no alteration in the severity of the illness. There were 11 patients treated with supportive therapy alone. No remissions occurred and each had early surgery.

In addition to supportive therapy, 12 patients received corticosteroid therapy. This was administered in the form of oral prednisone, 30 to $80 \mathrm{mg}$. daily, one patient also receiving prednisone enemata. Nine of these were already receiving the drug at the time of admission and three started treatment after 
admission. Corticosteroid treatment was initiated in special circumstances such as extreme youth, parental opposition to surgery, severe psychological disturbance preceding the illness, and language difficulties. It was effective in only one patient, a 6-year-old girl who rapidly improved 48 hours after beginning treatment. Further deterioration in the remaining 11 patients was arrested by surgery.

Operations were performed in 22 of the 23 patients at intervals of three to 43 days after admission. Corticosteroids were administered during the operation when there was a recent or past history of treatment with these drugs. Operation consisted of colectomy in 11 and procto-colectomy in 11 . Each patient had an ileostomy fashioned in the manner described by Brooke (1954). The type and duration of treatment before operation and the results of surgery are shown in Table II. The patients were divided into two groups which consisted of the 11 patients who had corticosteroid and supportive therapy and another 11 patients who had supportive therapy alone. The average time before operation was 21 days in the corticosteroid and supportive therapy group and 11 days in the group who had supportive therapy alone. Surgery was completed uneventfully in seven patients who had corticosteroid and supportive therapy but complications developed in the post-operative period in the remaining four. In one patient in whom permission for operation was not granted until the 43rd day, recovery was complicated by intestinal obstruction and unexplained post-operative collapse following a second laparotomy. This patient was one of two who experienced wound disruption; staphylococcal septicaemia developed in a third. The recovery of a patient with cirrhosis of the liver was complicated by the development of marked oedema.

Six of the 11 patients who had received supportive therapy alone before surgery made an uncom-

plicated recovery but five had post-operative complications from which two died. Death in the two patients resulted from peritonitis which led to severe electrolyte imbalance and the development of hepatic coma in one patient who had cirrhosis. Recovery was delayed in two by the development of a salt-losing renal state which responded to the administration of intravenous saline in conjunction with desoxycorticosterone. The illness in one of these two patients was further complicated by severe psychological disturbance. This complication was also present in the fifth patient.

Thus the results of surgery with prior supportive or supportive plus corticosteroid therapy were immediate recovery in 13 , delayed recovery in seven, and death in two. When the patient who recovered without operation is included, there were two deaths in the 23 patients, a mortality of $9 \%$.

\section{DISCUSSION}

Fulminant ulcerative colitis results from widespread involvement of the colon by ulcers which extend into the muscle coat. Warren and Sommers (1949) have described an arteritis of the submucosal vessels with this type of ulceration but an arteritis was not present in our material. The predilection of the ulcers for the muscular taeniae and the troughs between the transverse rugae, areas where the submucosa and mucosa are tightly bound to the muscle coat, has suggested to McGovern and Archer (1957) that inflammatory swelling in the troughs with extreme stretching in their depths produces a break in the mucosa so that extension through the submucosa results. Perforation is then liable to occur and the resultant peritonitis contributes in large part to the mortality of the illness.

The diagnosis of fulminant ulcerative colitis depends upon the clinical features, abnormal biochemical findings, and the sigmoidoscopic and radio-

TABLE II

TYPE OF MEDICAL TREATMENT AND RESULTS OF SURGERY

Steroid and Supportive Treatment

\begin{tabular}{ccl}
$\begin{array}{l}\text { Patient } \\
\text { Number }\end{array}$ & $\begin{array}{l}\text { Days to } \\
\text { Operation }\end{array}$ & Result \\
\hline 1 & 21 & Recovery \\
2 & 29 & Recovery \\
3 & 17 & Recovery \\
4 & 7 & Recovery \\
5 & 36 & Recovery \\
6 & 10 & Recovery \\
7 & 7 & Recovery \\
8 & 33 & Staphylococcal septicaemia; recovery \\
9 & 43 & Intestinal obstruction; post-operative \\
& & collapse; wound disruption; recovery \\
10 & 14 & Wound disruption; recovery \\
11 & 16 & Ascites; recovery
\end{tabular}

Supportive Treatment Alone

\begin{tabular}{lrl}
\hline $\begin{array}{l}\text { Patient } \\
\text { Number }\end{array}$ & $\begin{array}{l}\text { Days to } \\
\text { Operation }\end{array}$ & Result \\
\hline 12 & 3 & Recovery \\
13 & 5 & Recovery \\
14 & 18 & Recovery \\
15 & 17 & Recovery \\
16 & 10 & Recovery \\
17 & 12 & Recovery \\
18 & 17 & Salt-losing renal state; recovery \\
19 & 24 & Psychological disturbance; recovery \\
20 & 3 & Psychological disturbance; recovery \\
21 & 19 & Peritonitis; died \\
22 & 2 & Peritonitis; hepatic failure; died
\end{tabular}


logical findings we have described. We would stress the presence of fever, tachycardia, profuse fluid motions, mental disturbances, exquisite localized abdcminal tenderness, rebound tenderness, and increasing abdominal distension. The importance of anaemia, leucocytosis, hypoproteinaemia, hypoalbuminaemia, and falling serum sodium and potassium levels should also be stressed. Stools should be regularly inspected for increasing blood loss and collections should be accurately measured for volume and electrolyte content.

Sigmoidoscopy may help in identifying the disease as ulcerative colitis but is of limited help in diagnosing fulminant disease as deep ulceration is rarely seen. Occasionally, the examination is normal and an incorrect diagnosis of polyposis coli or carcinoma may follow the discovery of polyps.

Radiological examination has an important place in diagnosis, especially when the results of sigmoidoscopy are inconclusive. Plain $x$-ray examination of the abdomen is safe and is often of value in diagnosing fulminant colitis. If colonic dilatation is demonstrated in a known case of ulcerative colitis it always indicates fulminant disease. We have now seen this complication in 15 of 42 patients with fulminant colitis and deep ulceration extending into the muscle coat was present on each occasion. Dilatation has been attributed to potassium depletion (Cohn, Copit, and Tumen, 1956), to destruction of the myenteric plexuses (Bockus, Roth, Buchman, Kalser, Staub, Finklestein, and Valdes-Dapena, 1956), and to smooth muscle damage resulting from ulceration and inflammation spreading through the colonic wall (Sampson and Walker, 1961). None of these factors appears to explain all cases. Moreover, dilatation is frequently maximal in areas of the colon which are not severely diseased (Rankin et al., 1960). It is a sign of imminent perforation and in its presence barium enema examination is contraindicated. Barium enema examination in the absence of abdominal distension is useful in confirming the diagnosis of ulcerative colitis. Local irregularity of the colonic outline is at times very suggestive of extension of the ulcers into the muscle coat.

The recent improvement in our results may be attributed to intensive supportive therapy and the performance of early surgery. Supportive therapy is capable of bringing about a marked improvement in these patients and occasionally the desperately ill patient can improve to a point where surgery is attended by less risk. However, the often dramatic improvement that follows supportive therapy should be regarded as an early sign of a remission unless there is accompanying evidence of a decline in the severity of the diarrhoea and fever and tachycardia begin to abate.
Certain aspects, namely the provision of adequate protein, iron, sodium, and potassium require emphasis. The work of Steinfeld, Davidson, Gordon, and Greene (1960) has drawn attention to the protein loss from the gut in ulcerative colitis patients. Protein loss, as judged by progressive muscle wasting, was a striking feature of the illness in our patients and Falconer (1960) has shown that the losses may amount to $50 \mathrm{~g}$./day. We recommend that these patients be given at least this amount of albumin daily and feel that the problem of continual protein loss is an urgent reason for performing surgery when conservative treatment has failed to influence the course of the disease.

Beal, Skyring, McRae, and Firkin (1961) have shown that there is a surprising degree of iron depletion in ulcerative colitis patients, even when they are in clinical remission. The fulminant illness, especially when it arises in a patient with longstanding disease, will accentuate this problem. In one of our patients it was responsible for a marked degree of iron deficiency which persisted into the post-operative period. Treatment with iron should be begun early in the illness and serum iron levels should be checked before these patients are discharged from hospital.

Our experiences have also emphasized the degree of sodium and potassium depletion that exists in fulminant ulcerative colitis. Measurement of urinary excretion of sodium and potassium was a better guide to the degree of electrolyte depletion than concentrations in serum. For sodium replacement therapy $0.85 \%$ sodium chloride solutions were given but when sodium depletion persisted, sodium was given as $5 \%$ sodium chloride solution. Potassium was given intravenously by a slow infusion of $1.08 \%$ potassium chloride, and whenever possible was also given orally.

Whatever form of medical treatment is practised there is general agreement that it should not be unduly prolonged if it is not rapidly successful. We have previously expressed our preference for initial supportive therapy followed by early colectomy if no significant improvement occurred (Rankin et al., 1960). Others (Lennard-Jones and Vivian, 1960; Watkinson, 1961) recommend that corticosteroids be given a short trial in the hope of producing a remission. Corticosteroids have been shown to be of definite value in the treatment of less severe forms of ulcerative colitis in controlled trials (Truelove and Witts, 1955, 1959) but there is little evidence that they are of value in fulminant ulcerative colitis. We have previously cautioned against their use, although this opinion was formed at a time when supportive therapy, by current standards, was generally inadequate. Failure of corticosteroid 
treatment in the past may well have been due to insufficient attention to the needs of supportive therapy. Conclusions from our recent experience are therefore the more valid.

Corticosteroids in conjunction with supportive therapy were generally ineffective as there was only one remission in the 12 patients treated in this way. The longer interval before surgery in the majority of patients receiving corticosteroids was not the direct result of their receiving these drugs, as factors influencing the duration of conservative treatment, such as parental opposition to surgery and illness in the very young, were largely confined to this group. Moreover, only one patient had colonic dilatation which was regarded as an urgent indication for surgery whereas seven patients in the group who did not have corticosteroid therapy had this complication. There was no evidence that the use of corticosteroids was associated with increased morbidity and mortality in the patients undergoing operation. This finding is in agreement with the view of Ewart and Lennard-Jones (1960) who reviewed the illnesses in a large number of patients with all forms of ulcerative colitis undergoing surgery.

Corticosteroids in our experience have had little effect on the illness of the great majority of patients with fulminant ulcerative colitis. There is no indication for administering these drugs to patients with marked colonic dilatation in whom surgery should be carried out without delay for there is no way of telling whether perforation has already occurred. There seems to be little reason for not administering the drug to the remaining patients with fulminant ulcerative colitis although failure of previous corticosteroid therapy could be regarded as a contraindication, provided that treatment is not prolonged for longer than a week in the absence of marked improvement. Parenteral steroid therapy is essential for fulminant ulcerative colitis in patients undergoing surgery who have received the drug at any time previously.

Surgical treatment became necessary in 22 of the 23 patients we have described and it was performed at an average interval of 15 days after admission. Indications were failure to improve or sudden deterioration despite supportive or supportive and corticosteroid therapy. Marked dilatation of the colon, rebound tenderness, and recurrent hypotension from blood or fluid and electrolyte loss were regarded as urgent indications.

Most authors agree that one-stage colectomy with ileostomy is the operation of choice. Remarkable cessation of toxicity follows and fever and tachycardia disappear. It is our experience that the additional removal of the rectum is usually not essential. The only absolute indication is when deep ulceration is present in the rectum and marked haemorrhage has occurred. However, if the patient's general condition during operation is satisfactory, the rectum may be removed and the patient spared operation at a later date. We are not in favour of ileostomy alone unless the condition is so poor that no other procedure is justified. In most cases it will be possible to remove the colon if unsuccessful conservative treatment has not been unduly prolonged.

Our results can be described as satisfactory as there were two deaths in the 23 patients. This contrasts with the previous mortality of 11 deaths in 18 patients when surgery, if it was performed at all, was an heroic measure. The disadvantages of delaying surgery have been emphasized by Goligher (1961) who found 12 patients with open or closed perforation in a total of 41 patients. The absence of perforation in our patients encourages us to believe that it is the direct result of advising surgery early in the course of the illness.

Several important lessons can be learnt from a study of the two patients who died. One patient died from sepsis, including staphylococcal septicaemia. There was evidence to suggest that this was present at the time of the operation and it is a recognized hazard of prolonged intravenous therapy.

The second patient who died was a girl whose illness was complicated by chronic liver damage and portal venous obstruction. The hazards of the operation were anticipated but surgery gave the only possible chance of saving life. The paracolic gutters were entirely vascularized by anastomotic channels between the portal and systemic circulations. Ascitic fluid continued to escape around the ileostomy so that peritoneal soiling was unavoidable. It might be said that such a case should not be submitted to surgery but operation should not be rejected because the chances of success are poor.

Our management of the patient with fulminant ulcerative colitis continues along the following lines. An initial assessment of the severity of the illness is made and supportive therapy is begun in the manner outlined. Corticosteroid therapy is withheld when marked colonic dilatation is present and when it has been unsuccessful in the past. Early consultation with the surgeon follows and the anaesthetist is informed of the likelihood of surgery being performed. A daily assessment of the patient's condition is made, with particular attention to mental state, abdominal signs, and stool characteristics. If no improvement has occurred in a period of one to two weeks, colectomy is performed, or if urgent indications (such as colonic dilatation) appear, colectomy is performed as soon as it is practicable. Surgery is performed with a steroid cover when these drugs 
have been administered at any time previously. Satisfactory results can only be obtained in this illness when a team of physician, surgeon, and anaesthetist is responsible for management. Members of the team continue to have an important role in the post-operative period in assisting the patient to adjust to life with an ileostomy.

\section{SUMMARY}

This paper draws attention to the improved results that follow intensive medical treatment and early surgical intervention when medical treatment is unsuccessful. A reduction in mortality figures from $61 \%$ to $9 \%$ has been achieved.

Supportive treatment was begun at the earliest opportunity and particular attention was paid to replacing blood, protein, and fluid and electrolyte losses. There were no remissions in 11 patients who received supportive therapy alone and one remission in 13 patients who received additional corticosteroid therapy.

In our experience, corticosteroids have had no effect on the illness in the majority of patients. Past objections to corticosteroid therapy on the grounds that it postpones surgery, introduces complications, and delays post-operative recovery are no longer valid.

Surgery, consisting of colectomy and ileostomy, was necessary in 22 of the 23 patients. This was performed after an average period of 15 days' medical treatment. Urgent indications were colonic dilatations, rebound tenderness, and recurrent hypotension. Progressive deterioration was the indication in the remainder.
We wish to thank Drs. A. R. Cooke and A. P. Skyring for their help in the management of these patients and the honorary physicians who encouraged us to see those patients admitted under their care. The work of one of us (N.D.G.) was supported by the Bushell Trust.

\section{REFERENCES}

Beal, R. B., Skyring, A. P., McRae, J., and Firkin, B. G. (1961). The mechanism of production of anaemia in ulcerative colitis. Med. Res., 1, 7-8.

Bockus, H. L., Roth, J. L. A., Buchman, E., Kalser, M., Staub, W. R., Finklestein, A., and Valdes-Dapena, A. (1956). Life history of non-specific ulcerative colitis: relation of prognosis to anatomical and clinical varieties. Gastroenterologia (Basel), 86, 549-581.

Brooke, B. N. (1954). Ulcerative colitis and its surgical treatment. Livingstone, Edinburgh.

Cohn, E. M., Copit, P., and Tumen, H. J. (1956). Ulcerative colitis with hypopotassemia. Gastroenterology, 30, 950-957.

Ewart, W. B., and Lennard-Jones, J. E. (1960). Corticosteroids in preoperative medical management of ulcerative colitis. Do they affect surgical success? Lancet, 2, 60-64.

Falconer, C. W. A. (1960). Ulcerative colitis. J. roy. Coll. Surg. Edinb , 5, 269-286.

Goligher, J. C. (1961). Surgical treatment of ulcerative colitis. Brit. med. J., 1, 151-154.

Lennard-Jones, J. E., and Vivian, A. B. (1960). Fulminating ulcerative colitis-recent experience in management. Ibid., 2, 96-102.

McGovern, V. J., and Archer, G. T. (1957). The pathogenesis of ulcerative colitis. Aust. Ann. med., 6, 68-74.

Rankin, J. G., Goulston, S. J. M., Boden, R. W., and Morrow, A. W. (1960). Fulminant ulcerative colitis. Quart. J. Med., 29, 375-389.

Sampson, P. A., and Walker, F. C. (1961). Dilatation of the colon in ulcerative colitis. Brit. Med. J., 2, 1119-1123.

Steinfeld, J. L., Davidson, J. D., Gordon, R. S., and Greene, F. E (1960). The mechanism of hypoproteinemia in patients with regional enteritis and ulcerative colitis. Amer. J. Med., 29, 405-415.

Truelove, S. C., and Witts, L. J. (1955). Cortisone in ulcerative colitis. Brit. med. J., 2, 1041-1048.

- - (1959). Cortisone and corticotrophin in ulcerative colitis. Ibid., 1, 387-394

Warren, S., and Sommers, S. C. (1949). Pathogenesis of ulcerative colitis. Amer. J. Path., 25, 657-679.

Watkinson, G. (1961). Medical management of ulcerative colitis. Brit. med. J., 1, 147-151. 